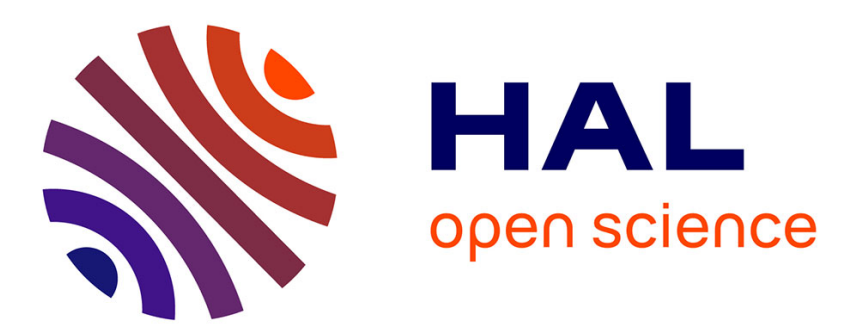

\title{
Net Neutrality Debate: Impact of Competition among ISPs
}

\author{
François Boussion, Patrick Maillé, Bruno Tuffin
}

\section{To cite this version:}

François Boussion, Patrick Maillé, Bruno Tuffin. Net Neutrality Debate: Impact of Competition among ISPs. COMSNETS 2012: 4th International Conference on Communication Systems and Networks, Jan 2012, Bangalore, India. pp.1-8. hal-00725489

\section{HAL Id: hal-00725489 \\ https://hal.science/hal-00725489}

Submitted on 27 Aug 2012

HAL is a multi-disciplinary open access archive for the deposit and dissemination of scientific research documents, whether they are published or not. The documents may come from teaching and research institutions in France or abroad, or from public or private research centers.
L'archive ouverte pluridisciplinaire HAL, est destinée au dépôt et à la diffusion de documents scientifiques de niveau recherche, publiés ou non, émanant des établissements d'enseignement et de recherche français ou étrangers, des laboratoires publics ou privés. 


\section{Net Neutrality Debate: Impact of Competition among ISPs}

\author{
François Boussion \\ ENS Cachan - Bretagne \\ Avenue Robert Schuman \\ 35170 Bruz - France \\ Email: francois.boussion@gmail.com \\ Patrick Maillé \\ Institut Telecom; Telecom Bretagne \\ 2, rue de la Châtaigneraie CS 17607 \\ 35576 Cesson-Sévigné Cedex, France \\ Email: patrick.maille@ telecom-bretagne.eu
}

\author{
Bruno Tuffin \\ INRIA Rennes Bretagne Atlantique \\ Campus de Beaulieu \\ 35042 Rennes Cedex, France \\ Email: bruno.tuffin@inria.fr
}

\begin{abstract}
Network neutrality has recently been the topic of an important debate, in both the telecommunication and political worlds, because of its potential impact in every-day life. While there has been many studies discussing the advantages and drawbacks of neutrality, there is no game-theoretical study dealing with the observable situation of competitive ISPs in front of a (quasi-)monopolistic content provider (CP), while it is a complaint from ISPs, and an illustration of the non-neutrality need. This paper provides a first game-theoretical analysis of relations between two competitive ISPs and a single $C P$, in the form of a four-level game, played at different time scales. This game is analyzed by backward induction. We show that while the complaint from ISPs is relevant with a such a competitive model, inserting side payments does not solve the problem.
\end{abstract}

\section{INTRODUCTION}

This paper deals with the network neutrality debate. The possibility of introducing non-neutrality comes from the increasing traffic asymmetry between Internet Service Providers (ISPs), mainly due to some prominent and resource consuming content providers $(\mathrm{CPs})$ which are usually connected to a single ISP. The typical example is YouTube (owned by Google), accessed by all users while hosted by a single Tier 1 ISP, and whose traffic now constitutes a non-negligible part of the whole Internet traffic. For this reason, there has been a surge of protest among ISPs, complaining that the current Internet business model where ISPs charge both endusers and content providers directly connected to them, and have public peering or transit agreements with other ISPs, is not relevant anymore, because they should charge content providers that are associated with other ISPs. This was first advocated at the end of 2005, by Ed Whitacre (CEO of AT\&T) [1]. The underlying concern is that investment is made by ISPs but content providers get an important part of the dividends. The revenue arising from online advertising (meaning showing graphical ads on regular web pages) is estimated at approximately a \$24 billion in 2009 [2] while textual ads on search pages has led to a combined revenue of $\$ 8.5$ billion in 2007 [3], those figures increasing every year. Meanwhile, transit prices - which constitutes the main source of revenues for transit ISPs - are decreasing. ISPs argue that there is no sufficient incentive for them to continue to invest on the network infrastructures if most benefits go to content providers. The threat is to lower the quality of service of
CPs that do not pay any fee to them, or even to block their traffic. This possibility has led to protests from CPs and user associations, complaining that this might impact the network development and is an impingement of freedom of speech [1]. This has launched a debate, essentially at the law and policy makers level, to decide whether the Internet should be neutral, i.e. all packets should receive equal treatments in terms of price and service. In the US, the Federal Trade Commission (FTC) released in 2007 a report not supporting neutrality constraints, increasing the debate at the political level. This debate is also active in Europe and in France, as illustrated by the open consultation on network neutrality launched in 2010. For instance, the French regulation authority, ARCEP, has published in its response a proposal intending to define how net neutrality could be implemented [4], [5].

Our goal here is to provide a mathematical analysis of the advantages and drawbacks of network neutrality. This type of work has recently received an increasing attention in the literature. The idea is to investigate the output of the interactions between selfish actors that are end users, CPs and ISPs, using the framework of non-cooperative game theory [6], [7].

Let us briefly describe here, non exhaustively, some important existing works in this direction. In [8], [9], the authors propose to share the revenue among providers using the Shapley value, the only mechanism that satisfies a set of axioms representing a sense of fairness; in this case CPs participate to the network access cost. The work in [10] analyzes how neutrality or non-neutrality affects provider investment incentives, network quality and user prices. A similar comparison is made in [11] between a two-sided pricing where ISPs are allowed to charge CPs, and one-sided pricing where such side-payments are not allowed. In each case, at the equilibrium of the game, the levels of investment in content and architecture are determined. The paper gives conditions on the ratio between parameters characterizing advertising rates and end-user price sensitivity, under which a non-neutral network outperforms a neutral one in terms of social welfare. On the other hand, [12] investigates the case where ISPs negotiate joint investment contracts with a $\mathrm{CP}$ in order to enhance the quality of service and increase industry profits. It is found that an unregulated regime leads to higher 
quality investments, but that ISPs have an incentive to degrade content quality. The paper [13] studies the implications of nonneutral behaviors, taking into account advertising revenues and considering both cooperative and non-cooperative scenarios. Finally, in [14], a game-theoretic model is considered with a single CP, a single ISP, a (consumers') demand function that depends on price and quality of service, and involving advertisement and network investment components.

In those works, there is in general a single ISP, and one or several CPs. Though, in practice, we often have ISPs in competition for customers, while for many services, the CPs are in a quasi monopole, a characteristic ISPs complain about. The typical example is YouTube. We propose to specifically address this issue in this paper. Remark that in addition to [11], considering competitive ISPs has been proposed in [15], but in their model, competition is over consumers, quality and prices for heterogenous CPs, none of those works consider a monopolistic $\mathrm{CP}$ as can be encountered for some applications.

The model we consider is inspired from [13], from which we borrow the basic assumptions, but to which we add the competition between ISPs. We analyze how this realistic assumption impacts the results of [13]. We propose a multilevel game where decisions are taken at different time scales. The solutions of the games at the largest time scales, played first, are determined using backward induction, meaning that players anticipate the impact on, and the resulting solution of, the games played later on at smaller time scales. We show that (Bertrand) competition among ISPs leads to zero revenues for them while the $\mathrm{CP}$ gets some, but that introducing side payments does not solve the problem.

The paper is organized as follows. Section II presents the basic assumptions of the model we are going to consider, the different levels of game and the mathematical description of the investigated comparison between the neutral and the non-neutral regimes. The next sections present the various game levels: Section III explains how users choose their ISP, depending on the price they have to pay; then we describe in Section IV how at a higher level the $\mathrm{CP}$, anticipating decisions of end users, chooses the content price. At an even higher level, still by backward induction, ISPs play a game on the access charge for end users; this Bertrand competition is described in Section V. We then describe the game at the highest level on the economic relationships between the ISPs and the CP, by determining the side payments of the CP to the ISPs in Section VI. We determine what happens if those prices are fixed by ISPs, based on a game; we also look at the case when they are decided by the $\mathrm{CP}$ or a regulator (maximizing social welfare for instance). Section VII concludes by discussing the impact and relevance of side payments on the providers' revenues. It shows that the initial idea to add revenue to the ISPs is not validated, and therefore that non-neutrality (by means of side payments) does not help, an argument in favor of neutrality sympathizers. We also give in this section directions for future research.

\section{MODEL}

We consider a single $\mathrm{CP}$, whose parameters will be indexed by 1 , and two ISPs, named (and indexed by) $A$ and $B$. The access price per unit of volume charged to users by the providers is $p_{1}$ for the $\mathrm{CP}$, and $p_{A}$ and $p_{B}$ for the two ISPs. In order to study non-neutrality, we also introduce side payments $q_{A}$ and $q_{B}$ representing the per unit of volume prices that the $\mathrm{CP}$ has to pay to $A$ and $B$, respectively. Remark here that we authorize $p_{A}, p_{B}, q_{A}$ and $q_{B}$ to potentially be negative, meaning that in that case, it is the ISPs who will actually pay the end users or the CP. The charges imposed by actors to other players are summarized in Figure 1, the arrows indicating the cash flows.

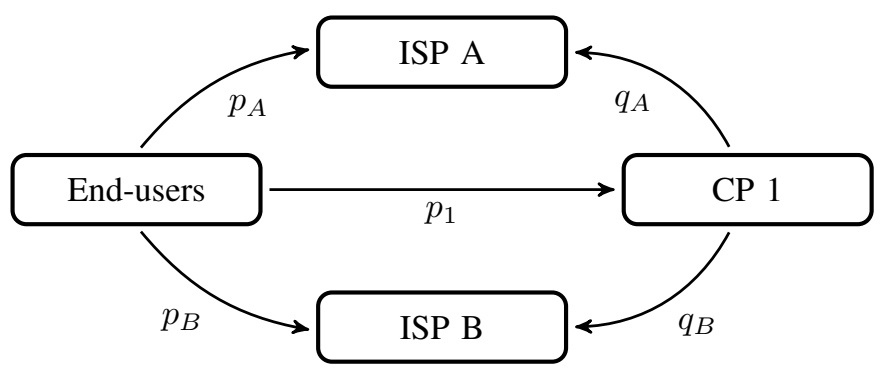

Fig. 1. Charging interactions between players

End users on the other hand are assumed infinitesimal, their total amount of traffic being modeled by a demand function, assumed to be linear and depending on the total price $\bar{p}$ that has to be paid to access the network:

$$
D:=\left[D_{0}-d \bar{p}\right]^{+}
$$

where $D_{0}$ is the total demand if the service were free, $d$ is the sensitivity to the price, and $x^{+}=\max (0, x)$ for all $x \in \mathbb{R}$. A clearer characterization of the total price $\bar{p}$ will be provided in the next section, but when going through ISP $A$ or $B$, the total price is respectively

$$
\begin{aligned}
\bar{p}_{A} & =p_{A}+p_{1} \\
\text { or } \quad \bar{p}_{B} & =p_{B}+p_{1} .
\end{aligned}
$$

The utilities (revenues) of the ISPs are given by the total amount of volume that goes through them times the total amount they get per unit:

$$
\begin{aligned}
& U_{A}=D_{A} \cdot\left(p_{A}+q_{A}\right) \\
& U_{B}=D_{B} \cdot\left(p_{B}+q_{B}\right) .
\end{aligned}
$$

The utility of the $\mathrm{CP}$ in this model is the sum of revenues gained by traffic flowing through $A$ and through $B$ (we do not look at advertisement revenues here, but it could be added without loss of generality by adding a per unit of volume revenue). It is thus given by

$$
U_{1}=D_{A}\left(p_{1}-q_{A}\right)+D_{B}\left(p_{1}-q_{B}\right) .
$$

The decision variables are the prices $p_{1}, p_{A}, p_{B}, q_{A}, q_{B}$, impacting end users (demand), as well as revenues of providers. 
Those variables are decided at different time scales or levels, that can be described as follows.

1) At the largest time scale, the side payments $q_{A}$ and $q_{B}$ are decided. In the neutral case, they are either fixed to 0 , or determined as a common value. They can be different in the non-neutral case, and can be determined either by the ISPs (in a game), the $\mathrm{CP}$, or a regulator. All those options will be investigated. Those determinations will be obtained anticipating the solution of the games below whatever the values of $q_{A}$ and $q_{B}$ (the so-called backward induction).

2) At a smaller time scale, for fixed values of $q_{A}$ and $q_{B}$, the ISPs fix their prices $p_{A}$ and $p_{B}$ during a noncooperative game to attract customers and maximize their revenues. Here again, the decisions are made anticipating the solutions at lower levels.

3) At an even smaller time scale, the $\mathrm{CP}$ sets the price $p_{1}$.

4) Finally, for those fixed values of $p_{1}, p_{A}, p_{B}, q_{A}, q_{B}$, the users choose their ISP (if not too expensive). This defines the user equilibrium.

All those interacting levels are now solved by backward induction, from the smallest time scale to the largest one.

\section{USER EQUILIBRIUM}

In order to define the user equilibrium, we assume that users choose the cheapest way to access content, i.e., that they choose the network with the smallest total price. According to Wardrop's principle [6], if $\bar{p}_{A}>\bar{p}_{B}$, then a user connected to $A$ would prefer to change ISPs, and reciprocally, if $\bar{p}_{A}>\bar{p}_{B}$ then a user connected to $B$ would switch to $A$. We therefore have

$$
\begin{gathered}
\bar{p}_{A}>\bar{p}_{B} \Rightarrow D_{A}=0 \\
\bar{p}_{B}>\bar{p}_{A} \Rightarrow D_{B}=0 .
\end{gathered}
$$

We define

$$
\bar{p}:=\min \left(\bar{p}_{A}, \bar{p}_{B}\right)
$$

as the total price via an ISP with strictly positive demand, i.e., the total price experienced by end-users.

The total demand $D$ can be decomposed into demand $D_{A}$ at $A$ and $D_{B}$ at $B$, such that

$$
D=D_{A}+D_{B}
$$

We have $D_{A}=D=D_{0}-d \bar{p}$ (respectively $D_{B}=D$ ) if $\bar{p}_{A}>\bar{p}_{B}$ (respectively $\bar{p}_{B}>\bar{p}_{A}$ ), but in the case when $\bar{p}_{A}=$ $\bar{p}_{B}=\bar{p}$ (i.e., $p_{A}=p_{B}$ ), we assume that there is a coefficient $\alpha \in[0,1]$ such that $D_{A}=\alpha D=\alpha\left(D_{0}-d \bar{p}\right)$ and $D_{B}=$ $(1-\alpha) D=(1-\alpha)\left(D_{0}-d \bar{p}\right)$. This parameter $\alpha$ represents the proportion of population going with $A$ because of some non-monetary preferences like the ISPs relative reputations.
We end up with the following set of equations to define the user equilibrium:

$$
\left\{\begin{aligned}
\bar{p}_{A} & =p_{A}+p_{1} \\
\bar{p}_{B} & =p_{B}+p_{1} \\
\bar{p} & =\min \left(\bar{p}_{A}, \bar{p}_{B}\right) \\
D & =D_{A}+D_{B} \\
D & =\left[D_{0}-d \bar{p}\right]^{+} \\
\bar{p}_{A}>\bar{p}_{B} & \Rightarrow D_{A}=0 \\
\bar{p}_{B}>\bar{p}_{A} & \Rightarrow D_{B}=0 \\
\bar{p}_{B}=\bar{p}_{A} & \Rightarrow D_{A}=\alpha D, D_{B}=(1-\alpha) D .
\end{aligned}\right.
$$

We have the following property:

Proposition 1. For each $\left(p_{A}, p_{B}\right)$, there exists a unique user equilibrium defining $D_{A}$ and $D_{B}$.

Proof: The proof of this proposition is obvious from the above equations: if $p_{A}>p_{B}, D_{A}=0, \bar{p}=p_{B}+p_{1}$ and $D_{B}=$ $D_{0}-d \bar{p}$; a symmetric characterisation is obtained $p_{B}>p_{A}$; while if $p_{A}=p_{B}, \bar{p}=\bar{p}_{A}=\bar{p}_{B}, D=\left[D_{0}-d \bar{p}\right]^{+}, D_{A}=\alpha D$ and $D_{B}=(1-\alpha) D$.

\section{CONTENT PROVIDER PRICE DETERMINATION}

The $\mathrm{CP}$ aims at maximizing his revenue $U_{1}$, for fixed values of $p_{A}, p_{B}, q_{A}, q_{B}$, making use of what the resulting user equilibrium will be. The problem is therefore $\max _{p_{1}} U_{1}$ with

$$
U_{1}=\left\{\begin{array}{cl}
{\left[D_{0}-d\left(p_{A}+p_{1}\right)\right]^{+}\left(p_{1}-q_{A}\right)} & \text { if } p_{A}<p_{B} \\
{\left[D_{0}-d\left(p+p_{1}\right)\right]^{+}} & \\
\times\left(p_{1}-\alpha q_{A}-(1-\alpha) q_{B}\right) & \text { if } p_{A}=p_{B}=p \\
{\left[D_{0}-d\left(p_{B}+p_{1}\right)\right]^{+}\left(p_{1}-q_{B}\right)} & \text { if } p_{A}>p_{B},
\end{array}\right.
$$

where we have plugged the user equilibrium as defined in the proof of Proposition 1 into the expression of $U_{1}$.

Differentiating with respect to $p_{1}$ in all three cases, we get for prices such that $\min \left(p_{A}, p_{B}\right)+p_{1}<D_{0} / d$,

$\frac{\partial U_{1}}{\partial p_{1}}=\left\{\begin{aligned} D_{0}-d\left(p_{A}+p_{1}\right)-d\left(p_{1}-q_{A}\right) & \text { if } p_{A}<p_{B} \\ D_{0}-d\left(p+p_{1}\right) & \\ -d\left(p_{1}-\alpha q_{A}-(1-\alpha) q_{B}\right) & \text { if } p_{A}=p_{B}=p \\ D_{0}-d\left(p_{B}+p_{1}\right)-d\left(p_{1}-q_{B}\right) & \text { if } p_{A}>p_{B},\end{aligned}\right.$

and the maximum of $U_{1}$ is therefore obtained at

$$
p_{1}^{*}=\left\{\begin{array}{cl}
\frac{1}{2}\left(\frac{D_{0}}{d}+q_{A}-p_{A}\right) & \text { if } p_{A}<p_{B} \\
\frac{1}{2}\left(\frac{D_{0}}{d}+\alpha\left(q_{A}-p\right)\right. & \\
\left.+(1-\alpha)\left(q_{B}-p\right)\right) & \text { if } p_{A}=p_{B}=p \\
\frac{1}{2}\left(\frac{D_{0}}{d}+q_{B}-p_{B}\right) & \text { if } p_{A}>p_{B} .
\end{array}\right.
$$

Note that the following condition must be met for this price to lead to positive demands:

$$
\begin{cases}\frac{D_{0}}{d}>p_{A}+q_{A} & \text { if } p_{A}<p_{B} \\ \frac{D_{0}}{d}>p+\alpha q_{A}+(1-\alpha) q_{B} & \text { if } p_{A}=p_{B}=p \\ \frac{D_{0}}{d}>p_{B}+q_{B} & \text { if } p_{A}>p_{B} .\end{cases}
$$

That same condition also ensures that the $\mathrm{CP}$ makes a strictly positive profit.

Not surprisingly, the price $p_{1}^{*}$ increases when the demand sensitivity $d$ decreases and when the side payments increase. 


\section{Pricing Game Between ISPS: Bertrand COMPETITION}

Before the users decide which ISP to join and the $\mathrm{CP}$ chooses $p_{1}$, the ISPs play a pricing game, making use of what the CP and users decisions would be. In order to determine the solution of this game, we need to express the utility functions of the ISPs. Replacing in $U_{A}$ and $U_{B}$ the expression $p_{1}$ by $p_{1}^{*}$ in (5), and using the user equilibrium in the proof of Proposition 1, we get

$U_{A}=\left\{\begin{aligned} \frac{1}{2}\left[D_{0}-\left(p_{A}+q_{A}\right) d\right]\left(p_{A}+q_{A}\right) & \text { if } p_{A}<p_{B} \\ \frac{\alpha}{2}\left(D_{0}-\left(p+\alpha q_{A}+(1-\alpha) q_{B}\right) d\right) & \\ \times\left(p+q_{A}\right) & \text { if } p_{A}=p_{B}=p \\ 0 & \text { if } p_{A}>p_{B} .\end{aligned}\right.$

We get a similar expression for $U_{B}$, just inverting the indexes $A$ and $B$, and changing $\alpha$ to $1-\alpha$.

To determine if there is a Nash equilibrium to this pricing game, we need to determine the best-response curves of ISPs. Recall (see [7]) that a Nash equilibrium would be a price profile $\left(p_{A}, p_{B}\right)$ such that no ISP can improve his utility by unilaterally changing his price. The best-response curves are defined as (by expliciting the dependence of $U_{A}$ and $U_{B}$ on $\left.p_{A}, p_{B}\right)$

$$
\begin{aligned}
& \operatorname{BR}_{A}\left(p_{B}\right)=\arg \max _{p_{A} \geq 0} U_{A}\left(p_{A}, p_{B}\right) \text { and } \\
& \operatorname{BR}_{B}\left(p_{A}\right)=\arg \max _{p_{B} \geq 0} U_{B}\left(p_{A}, p_{B}\right) .
\end{aligned}
$$

With those notations, a Nash equilibrium is a point $\left(p_{A}^{*}, p_{B}^{*}\right)$ for which $\mathrm{BR}_{A}\left(p_{B}^{*}\right)=p_{A}^{*}$ and $\mathrm{BR}_{B}\left(p_{A}^{*}\right)=p_{B}^{*}$. Graphically, if we draw the two best-response curves on the same figure, the set of Nash equilibria is then the (possibly empty) set of intersection points of those curves.

We also assume throughout the paper that there is a discretization value $\epsilon$ on the price range values. Indeed, the prices are usually defined through a unit (cents for instance) such that when defining a price difference, this cannot be less than $\epsilon$.

We have the following proposition:

Proposition 2. Assuming an $\epsilon$ close enough to zero, there is a unique equilibrium $\left(p_{A}^{*}, p_{B}^{*}\right)$ to the price war:

1) If $q_{A}<q_{B}$, the equilibrium is $\left(-q_{A},-q_{A}-\epsilon\right)$,

2) If $q_{A}>q_{B}$, the equilibrium is $\left(-q_{B}-\epsilon,-q_{B}\right)$,

3) If $q_{A}=q_{B}=q$, the equilibrium is $(-q,-q)$.

We typically end up with a Bertrand competition [7], in which ISPs decrease their price to attract all demand from the competitor, up to the moment where revenue becomes zero for one of them. In this case, when the side payments $q_{A}$ and $q_{B}$ are positive, then because of the competition it is the ISPs which give money to the end users, the money they are getting coming from the CP. Best-response curves (and the Nash equilibrium) are illustrated by Figure 2 when $q_{A}<q_{B}$ (the case $q_{B}<q_{A}$ is symmetric), and in Figure 3 when $q_{A}=$ $q_{B}$.

Proof: We start by determining the best-response functions of ISPs. We do it for $A$, as the case of $B$ is symmetric. We can note that $U_{A}$ in (7), as a function of $p_{A}$ with $p_{B}$

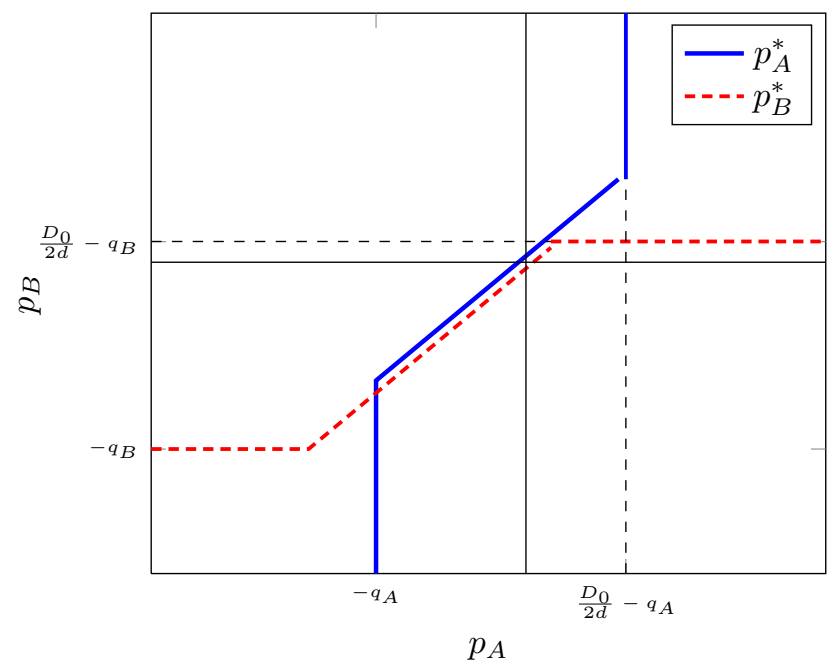

Fig. 2. best-response curves in the price war when $q_{A}<q_{B}$. Parameter values: $q_{A}=20, q_{B}=30, D_{0}=100, d=2, \alpha=0.5$.

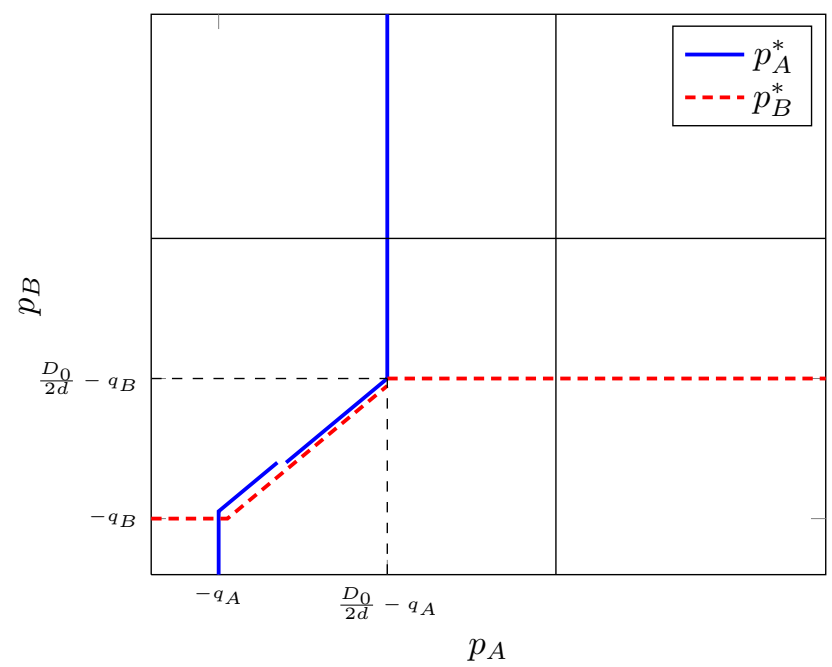

Fig. 3. Best-response curves in the price war when $q_{A}=q_{B}$. Parameter values: $q_{A}=q_{B}=50, D_{0}=100, d=2, \alpha=0.4$.

fixed, is continuous on $\left(-\infty, p_{B}\right)$, has a given value on $p_{B}$, and is 0 on $\left(p_{B},+\infty\right)$. The function of $p_{A}$ that defines $U_{A}$ for $p_{A}<p_{B}, \frac{1}{2}\left[D_{0}-\left(p_{A}+q_{A}\right) d\right]^{+}\left(p_{A}+q_{A}\right)$, is first increasing up to $\frac{D_{0}}{2 d}-q_{A}$, then decreasing up to $\frac{D_{0}}{d}-q_{A}$, and then constant equal to 0 . Remark therefore that for $A$ to obtain a strictly positive demand (and thus, utility), the condition in (6) must be satisfied, i.e., the $\mathrm{CP}$ also makes some benefit.

As a consequence:

- if $\frac{D_{0}}{2 d}-q_{A}<p_{B}$, the maximum of $U_{A}$ for $p_{A}<p_{B}$ is obtained at that point, and equals $\frac{1}{8} \frac{D_{0}^{2}}{d}$.

- if $-q_{A} \leq p_{B} \leq \frac{D_{0}}{2 d}-q_{A}$, then the maximum of $U_{A}$ for $p_{A}<p_{B}$ is obtained at $p_{B}-\epsilon$ and is $\frac{1}{2}\left(D_{0}-\left(\left(p_{B}-\right.\right.\right.$ $\left.\left.\epsilon)+q_{A}\right) d\right)\left(\left(p_{B}-\epsilon\right)+q_{A}\right)$.

- if $p_{B}<-q_{A}$ then $p_{A}<p_{B}$ would imply that the revenue 
of $A$ cannot be positive. It is therefore better for $A$ to get zero revenue, for example with the price $p_{A}=-q_{A}$, but actually any price $p_{A}>p_{B}$ is a best-reply of $A$ in that case.

To determine the best-reply on $(-\infty,+\infty)$, we now only have to compare the value at those points with $U_{A}\left(p_{B}, p_{B}\right)$, which gives:

- when $p_{B}>D_{0} /(2 d)-q_{A}, U_{A}\left(\frac{D_{0}}{2 d}-q_{A}, p_{B}\right)=\frac{1}{8} \frac{D_{0}^{2}}{d}$ to be compared with $U_{A}\left(p_{B}, p_{B}\right)=\frac{\alpha}{2}\left[D_{0}-\left(p_{B}+\alpha q_{A}+\right.\right.$ $\left.\left.(1-\alpha) q_{B}\right) d\right]^{+}\left(p_{B}+q_{A}\right)$. We prove here that it is always better for $A$ to set $p_{A}=\frac{D_{0}}{2 d}-q_{A}$ in that case.

- When $p_{B}>D_{0} / d-\left(\alpha q_{A}+(1-\alpha) q_{B}\right)$ then $U_{A}\left(p_{B}, p_{B}\right)=0$ and player $A$ is better off setting $p_{A}=D_{0} /(2 d)-q_{A}$.

- Otherwise, the difference $U_{A}\left(D_{0} /(2 d)-q_{A}, p_{B}\right)$ $U_{A}\left(p_{B}, p_{B}\right)$ can be seen as a degree-2 polynomial in $D_{0}$, with discriminant of the same sign as

$$
-\alpha(1-\alpha)\left(p_{B}+q_{A}\right)\left(p_{B}+q_{B}\right) .
$$

We have $p_{B}>D_{0} /(2 d)-q_{A}$ therefore $p_{B}+q_{A}>0$. On the other hand, Provider $B$ should set $p_{B} \geq-q_{B}$ in order to make profit, so that the discriminant is non-positive, and thus the difference $U_{A}\left(D_{0} /(2 d)-\right.$ $\left.q_{A}, p_{B}\right)-U_{A}\left(p_{B}, p_{B}\right)$ is always non-negative.

- When $-q_{A} \leq p_{B} \leq D_{0} /(2 d)-q_{A}, U_{A}\left(p_{B}-\epsilon, p_{B}\right)=$ $\frac{1}{2}\left[D_{0}-\left(p_{B}-\epsilon+q_{A}\right) d\right]\left(p_{B}-\epsilon+q_{A}\right)$, still to be compared with $U_{A}\left(p_{B}, p_{B}\right)=\frac{\alpha}{2}\left[D_{0}-\left(p_{B}+\alpha q_{A}+\right.\right.$ $\left.\left.(1-\alpha) q_{B}\right) d\right]^{+}\left(p_{B}+q_{A}\right)$. In that case, Provider $A$ is better off setting $p_{A}=p_{B}$ when $p_{B}$ is above a threshold, and setting $p_{A}=p_{B}-\epsilon$ otherwise. When $\epsilon \rightarrow 0$ that threshold equals $\frac{D_{0}}{d}-q_{A}-\alpha\left(q_{A}-q_{B}\right)$.

- the case $p_{B}<-q_{A}$ does not offer any possibility for $A$ to have a positive revenue, so does not need to be considered here.

A symmetric behaviour is deduced for $B$.

On Figure 3, it can be noted (looking at the blue curve) that when $p_{B}$ is right above $-q_{A}$, the best response for $B$ is $p_{B}-\epsilon$, but becomes $p_{B}$ when when $p_{B}$ increases; this comes from the comparison between $U_{A}\left(p_{B}, p_{B}\right)$ and $U_{A}\left(p_{B}-\epsilon, p_{B}\right)$ described above.

But remark that we cannot have simultaneously the maximum obtained at the value of the opponent, i.e., $\mathrm{BR}_{A}\left(p_{B}\right)=$ $\operatorname{BR}_{B}\left(p_{A}\right)=p_{A}=p_{B}=p$, with strictly positive utilities. Indeed, it is not possible that each utility value at the price of the opponent is larger than the value just below, i.e., that

$$
\left\{\begin{aligned}
\lim _{p_{A} \rightarrow p} U_{A}\left(p_{A}, p\right) \leq U_{A}(p, p) \\
\lim _{p_{B} \rightarrow p} U_{B}\left(p, p_{B}\right) \leq U_{B}(p, p) .
\end{aligned}\right.
$$

For strictly positive utilities, this would be equivalent to

$$
\begin{cases}D_{0}-\left(p+q_{A}\right) d \leq \alpha\left(D_{0}-\left(p+\alpha q_{A}+(1-\alpha) q_{B}\right) d\right) \\ D_{0}-\left(p+q_{B}\right) d \leq(1-\alpha)\left(D_{0}-\left(p+\alpha q_{A}+(1-\alpha) q_{B}\right) d\right),\end{cases}
$$

i.e.,

$$
\left\{\begin{array}{l}
\frac{D_{0}}{d}-p \leq q_{A}+\alpha\left(q_{A}-q_{B}\right) \\
\frac{D_{0}}{d}-p \leq q_{B}+(1-\alpha)\left(q_{B}-q_{A}\right) .
\end{array}\right.
$$

Summing the two equations, this leads to

$$
\frac{D_{0}}{d}-p \leq \alpha q_{A}+(1-\alpha) q_{B},
$$

which contradicts the fact that demand at $p$ is positive (and therefore the utilities $U_{A}(p, p)$ and $U_{B}(p, p)$ ), using $p_{1}^{*}$ in (5).

As a consequence, and as shown in Figures 2 and 3, a player's best interest is always to play less than its opponent, up to the moment when the revenue of one of them becomes zero (which means by (7) that $p_{A}=-q_{A}$ or $p_{B}=-q_{B}$ ). In that case, if $q_{A}=q_{B}$, we get the third item in the proposition, no ISP can reduce his price anymore, while if $q_{A}<q_{B}, B$ can reduce his price to $-q_{A}-\epsilon$, while $A$ cannot go below $-q_{A}$ without having a negative revenue. In that situation, no ISP can unilaterally increase his revenue. The symmetric case $q_{A}>q_{B}$ is obtained similarly. This concludes the proof of the proposition.

\section{SIDE PAYMENTS DETERMINATION}

We consider at the higher level three possibilities for the choice of side payments $q_{A}$ and $q_{B}$. We first look at the case when they are determined by the CP (even if unlikely in practice), then the case when they result from a game played between ISPs, and finally the case when they are determined by a regulator (to maximize social welfare).

\section{A. Determined by the $C P$}

Plugging into the expression of the utility $U_{1}$ of the CP (4) the optimal value $p_{1}^{*}$ in (5) and the prices of the war determined in Proposition 2, we get

$U_{1}\left(q_{A}, q_{B}\right)= \begin{cases}\frac{1}{4 d}\left(D_{0}-d\left(q_{B}-q_{A}-\epsilon\right)\right)^{2} & \text { if } q_{A}<q_{B} \\ \frac{1}{4} \frac{D_{0}^{2}}{d} & \text { if } q_{A}=q_{B} \\ \frac{1}{4 d}\left(D_{0}-d\left(q_{A}-q_{B}-\epsilon\right)\right)^{2} & \text { if } q_{A}>q_{B}\end{cases}$

If the parameter $\epsilon$ is a very small amount (less than $\left|q_{A}-q_{B}\right|$, if it is positive), the optimum is obtained when $q_{A}=q_{B}=q$, irrespective of its value because an increase of side payments would result in an equal increase of the value $p_{1}^{*}$, so that the revenue $U_{1}$ does not change. Remark that the ISPs then make no profit at all, all benefits going to the $\mathrm{CP}$ with a total value $\frac{D_{0}^{2}}{4 d}$.

\section{B. Determined by the ISPs, through a game}

If we instead assume that the side payments are noncooperatively determined by the ISPs, recall first that the utility functions of $A$ and $B$ are (using the equilibrium of 
Proposition 2, highlighting the dependence on $q_{A}$ and $q_{B}$ ):

$$
\begin{aligned}
& U_{A}\left(q_{A}, q_{B}\right)=\left\{\begin{aligned}
\frac{1}{2}\left[D_{0}-\left(q_{A}-q_{B}-\epsilon\right) d\right] & \text { if } q_{A}>q_{B} \\
\times\left(q_{A}-q_{B}-\epsilon\right) & \text { if } q_{A}=q_{B}=q \\
0 & \text { if } q_{A}<q_{B},
\end{aligned} \quad\right. \\
& U_{B}\left(q_{A}, q_{B}\right)=\left\{\begin{aligned}
& \frac{1}{2}\left[D_{0}-\left(q_{B}-q_{A}-\epsilon\right) d\right] \\
& \times\left(q_{B}-q_{A}-\epsilon\right) \text { if } q_{B}>q_{A} \\
& 0 \text { if } q_{A}=q_{B}={ }_{q} \\
& 0 \text { if } q_{B}<q_{A} .
\end{aligned}\right.
\end{aligned}
$$

In that case, the ISPs best interest is always to play a larger side payment than his opponent. As a consequence, there is no Nash equilibrium, the side payments will naturally tend to $+\infty$.

Remark here too that sending the side payments to infinity does not mean that the perceived price goes itself to infinity (nor that demand is 0), those payments are indeed given back to the end users by the ISPs, and the content providers price is also increased by this amount (nullifying the effect).

\section{Determined by a regulator}

A regulator can either decide to maximize the revenue of the supply chain (sum of utilities of the ISPs plus the CP), the user welfare (end-users surplus), or the social welfare (including user welfare and all providers utilities).

In our model, all subscribers pay the same unit price $\bar{p}=p_{1}+\min \left(p_{A}, p_{B}\right)$. But some of them would have accepted (or were willing) to pay more than $\bar{p}$ to benefit from the service: for example among the total demand $D(\bar{p})$, the amount of traffic that was (unitarily) worth at least $\tilde{p}$ is $D(\tilde{p})$. The difference between the actual value of the traffic (the price the user is willing to pay) and the price actually paid is considered to be a profit made by the user. User Welfare (UW) is defined as the total profit of the users corresponding to the service. It can be computed using the demand function as

$$
\mathrm{UW}=\int_{p=\bar{p}}^{+\infty} D(p) d p,
$$

and is illustrated in Figure 4. For our linear demand function, we have

$$
\mathrm{UW}=\frac{d}{2}\left(\frac{D_{0}}{d}-\bar{p}\right)^{2}
$$

when $\bar{p} \in\left[0, D_{0} / d\right]$. UW naturally increases when the perceived price decreases, because new subscribers get some surplus and the already present subscribers experience a higher surplus.

Similarly, the total price paid by users equals $\bar{p} D(\bar{p})$. This amount is shared among the three providers, and is therefore the total value of the supply chain. Its expression is simply

$$
U_{1}+U_{A}+U_{B}=\bar{p}\left[D_{0}-d \bar{p}\right]^{+}
$$

as illustrated in Figure 4.

Finally, the Social Welfare is defined as the overall value of the service for the society. It therefore includes the surpluses

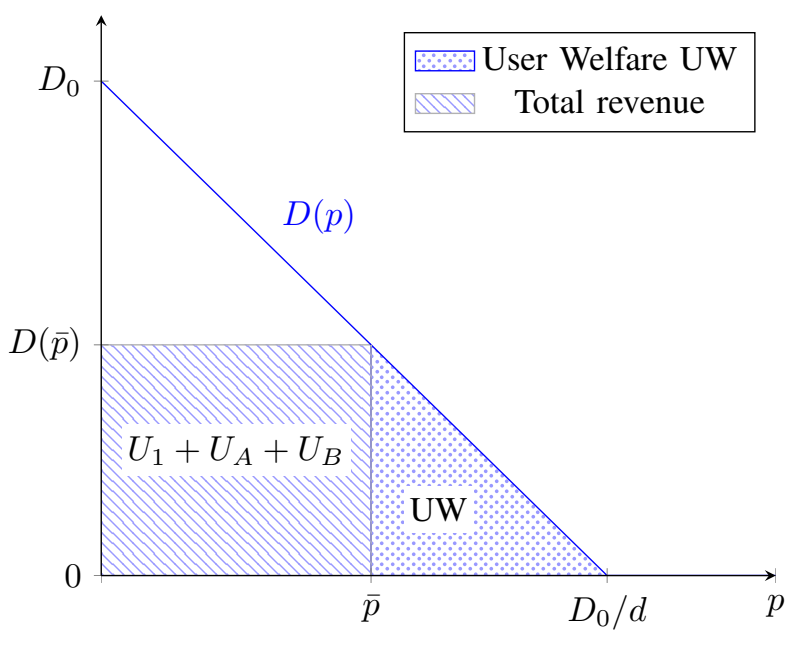

Fig. 4. The different surpluses

of all actors, and equals

$$
\mathrm{SW}=U_{1}+U_{A}+U_{B}+\mathrm{UW} .
$$

For the linear demand function $D$, we have

$$
\mathrm{SW}=\frac{1}{2 d}\left(D_{0}^{2}-d^{2} \bar{p}^{2}\right) .
$$

In summary, Social Welfare corresponds to the total value that the service has for subscribers, without considering any monetary exchanges because they stay within the society. It is therefore natural that Social Welfare increases when demand increases, i.e. when the perceived price decreases.

As Figure 4 illustrates, all the measures defined in this section (total provider surplus, user welfare, social welfare) depend only on the perceived price $\bar{p}$ that results from the pricing decisions of the $\mathrm{CP}$ and the ISPs. The following proposition gives the value of that perceived price when the side payments vary.

Proposition 3. When $\epsilon$ tends to zero, at the overall pricing equilibrium (including $C P$ and ISPs decisions) the perceived price $\bar{p}=p_{1}^{*}+\min \left(p_{A}, p_{B}\right)$ tends to

$$
\bar{p}=\frac{1}{2}\left(\frac{D_{0}}{d}+\left|q_{A}-q_{B}\right|\right) .
$$

Proof: We directly apply Proposition 2:

- if $q_{A}<q_{B}$ then the equilibrium of the pricing game is $\left(p_{A}, p_{B}\right)=\left(-q_{A},-q_{A}-\epsilon\right)$. Since $p_{B}<p_{A}$, the CP decision gives $p_{1}^{*}=\frac{1}{2}\left(D_{0} / d+q_{B}+q_{A}+\epsilon\right)$ from (5), which yields $\bar{p}=\frac{1}{2}\left(\frac{D_{0}}{d}+\left|q_{A}-q_{B}\right|+\epsilon\right)$ if $\epsilon<\mid q_{A}-$ $q_{B} \mid$. When $\epsilon \rightarrow 0$ we obtain (12).

- The case $q_{A}>q_{B}$ can be treated similarly.

- If $q_{A}=q_{B}=q$, then $p_{A}=p_{B}=-q$ from Proposition 2, and (5) implies that $p_{1}^{*}=\frac{1}{2}\left(D_{0} / d+2 q\right)$. Then $\bar{p}=\frac{D_{0}}{2 d}$, which is still consistent with (12). 
1) Side-payments to maximize the supply chain value: Summing the utilities (8), (9), and (10) of the supply chain, we get

$U_{1}+U_{A}+U_{B}= \begin{cases}\left.\frac{D_{0}^{2}}{4 d}-\frac{d}{4}\left(\left|q_{A}-q_{B}\right|-\epsilon\right)\right)^{2} & \text { if } q_{A} \neq q_{B} \\ \frac{D_{0}^{2}}{4 d} & \text { if } q_{A}=q_{B},\end{cases}$

which is maximized when $q_{A}=q_{B}=q$ and does not depend on the value of $q$. Note that the value $\frac{1}{4} \frac{D_{0}^{2}}{d}$ is actually the absolute maximum that could be reached by all providers if they decided to collaborate: this can be easily seen on Figure 4, since $\frac{1}{4} \frac{D_{0}^{2}}{d}$ is the surface of the largest rectangle below the demand function.

2) Side payments to maximize User Welfare or Social Welfare: If the objective of a regulator is to maximize user or social welfare, as we saw in the beginning of this section the side payments should be chosen so as to minimize the resulting perceived price $\bar{p}$. From Proposition 3, that perceived price is minimized when $q_{A}=q_{B}=q$, for any value of $q$.

3) On the efficiency of side payments: The previous subsections have highlighted the fact that equal side payments are likely to be selected. On the other hand, recall that when $q_{A}=q_{B}$ then no ISP makes any profit, and the CP takes all the supply chain surplus, that equals $\frac{D_{0}^{2}}{4 d}$. This suggests that the ISPs' arguments regarding the CPs extracting most of the benefit is legitimate. However, our model also suggests that side payments would not benefit to ISPs, since equal side payments have no effect while asymmetric side payments imply that one ISP is thrown out of the market.

\section{DISCUSSION, CONCLUSIONS AND FUTURE WORK}

We have provided in this paper a model describing the interactions between two ISPs in competition, a CP and end users connecting to the network through the least expensive ISP. With respect to the literature, we believe that considering competitive ISPs and a single CP is a more realistic representation of the current network where we have a quasi-monopole for some applications (for instance YouTube) while several ISPs are in competition (an argument of ISPs). The goal is to study the impact of side payments on providers' revenues and conclude if this can help ISPs to increase their revenue, as they claim in the current network neutrality debate.

The above sections have presented a four-level game where (from the largest to the shortest time scale) the side payments are first determined, then a pricing game is played between ISPs, followed by the content provider price, and finally, knowing all those prices, end users choose their ISP, or none if too expensive. All those levels are played by backward induction, meaning that players anticipate the solutions of the later games when choosing their strategies.

The following remarks can be made from our analysis:

- The idea of introducing side payments in the net neutrality debate was to ensure that ISPs would recover their cost and reinvest in the architecture but, as we have shown, this does not help the ISPs to get a higher revenue. If ISPs choose the side payments, there is no equilibrium and even in other cases, the equilibrium is independent of those side payments;

- the reason for that is the Bertrand competition between ISPs which drives their revenue to zero; side payments are "counter-balanced" by competition in the sense that side payments are poured back to end users in order to attract them.

As a conclusion, side payments are actually not of interest for ISPs, they do not help in a competitive situation.

As future research, we would like to go into several directions: first to include several CPs with different contents, but such that some end users are targeting only a subset of them, for all possible subsets. ISPs may also charge each other to let the CPs not connected to them reach their own customers. Other extensions to our model include dealing with a nonlinear demand, including a congestion cost as in [16] (but where it is not related to network neutrality), and/or including architecture investment and content innovation characteristics, for the ISPs and the CP respectively.

\section{ACKNOWLEGMENTS}

This work has been supported by INRIA's ARC project MENEUR on network neutrality and French ANR VERSO project CAPTURES on competition beween providers.

\section{REFERENCES}

[1] A. Odlyzko, "Network neutrality, search neutrality, and the never-ending conflict between efficiency and fairness in markets," Review of Network Economics, vol. 8, no. 1, pp. 40-60, 2009.

[2] A. Ghosh and A. Sayedi, "Expressive auctions for externalities in online advertising," in Proc. of 19th World Wide Web conference (WWW), Raleigh, NC, USA, April 2010.

[3] D. Liu, J. Chen, and A. Whinston, "Competing keyword auctions," in Proc. of 4th Workshop on Ad Auctions, Chicago, IL, USA, Jul 2008.

[4] ARCEP, "Neutralité de l'internet et des réseaux: propositions et orientations," 2010, http://www.arcep.fr/uploads/tx_gspublication/ net-neutralite-orientations-sept2010.pdf.

[5] S. Wong, R.-M. J., and E. Altman, "Public consultations on Net Neutrality 2010: USA, EU and France," SSRN, Tech. Rep., 2010, http://papers.ssrn.com/sol3/papers.cfm?abstract_id=1684086.

[6] D. Fudenberg and J. Tirole, Game Theory. MIT Press, Cambridge, Massachusetts, 1991.

[7] M. Osborne and A. Rubinstein, A Course in Game theory. MIT Press, 1994.

[8] R. Ma, D.-M. Chiu, J. Lui, V. Misra, and D. Rubenstein, "Interconnecting eyeballs to content: A Shapley value perspective on isp peering and settlement," in Proc. of International Workshop on Economics of Networked Systems (NetEcon), 2008, pp. 61-66.

[9] —, "On cooperative settlement between content, transit and eyeball internet service providers," in Proc. of ACM International Conference on Emerging Networking EXperiments and Technologies (CoNEXT), 2008.

[10] J. Musacchio, J. Walrand, and G. Schwartz, "Network neutrality and provider investment incentives," in Proc. of 41st Asilomar Conference on Signals, Systems and Computers (ACSSC), Nov 2007, pp. 1437 1444.

[11] J. Musacchio, G. Schwartz, and J. Walrand, "A two-sided market analysis of provider investment incentives with an application to the net-neutrality issue," Review of Network Economics, vol. 8, no. 1, 2009.

[12] C. Saavedra, "Bargaining power and the net neutrality debate," 2009, sites.google.com/site/claudiasaavedra/attachments/bargaining_power. pdf.

[13] E. Altman, P. Bernhard, S. Caron, G. Kesidis, J. Rojas-Mora, and S. Wong, "A study of non-neutral networks with usage-based prices," in Proc. of 3rd ETM Workshop, Amsterdam, The Netherlands, Sept 2010. 
[14] E. Altman, A. Legout, and Y. Xu, "Network non-neutrality debate: An economic analysis," in Proc. of Networking. Barcelona, Spain: LNCS 6641, Springer Verlag, May 2011, pp. 68-81.

[15] P. Njoroge, A. Ozdaglar, N. Stier-Moses, and G. Weintraub, "Investment in two sided markets and the net neutrality debate," Columbia University, Decision, Risk and Operations Working Papers Series, Tech. Rep. DRO2010-05, 2010.

[16] D. Acemoglu and A. Ozdaglar, "Competition and efficiency in congested markets," Mathematics of Operations Research, vol. 32, no. 1, pp. 1-31, Feb 2007. 